\title{
Charge Loss in Gas-Phase Multiply Negatively Charged Oligonucleotides
}

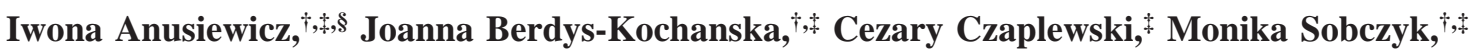 \\ Emma M. Daranowski,, Piotr Skurski,,+ \\ Chemistry Department and Henry Eyring Center for Theoretical Chemistry, University of Utah, Salt Lake City, \\ Utah 84112, and Department of Chemistry, University of Gdansk, 80-952 Gdansk, Poland
}

Received: July 12, 2004; In Final Form: October 20, 2004

\begin{abstract}
In an attempt to shed light on the mechanism by which gaseous samples of negatively charged oligonucleotides undergo extremely slow (i.e., over 1-1000 s) charge loss, we have carried out molecular dynamics simulations on an oligonucleotide anion, $\mathrm{T}_{5}{ }^{3-}$, containing five thymine, deoxyribose, and phosphate units in which the first, third, and fifth phosphates are negatively charged. The study is aimed at determining the rate at which an electron is detached from such a trianion by way of an internal Coulomb repulsion induced event. In this process, the intrinsic 5.0-5.1 eV electron binding strength of each phosphate site is reduced by the repulsive Coulomb potentials of the other two negative sites. As geometrical fluctuations cause the distances among the three negative phosphate sites to change, this causes the Coulomb repulsion energies at these sites to fluctuate. Once the Coulomb potential at any phosphate site exceeds ca. $5 \mathrm{eV}$, the electron on that site is able to undergo autodetachment. Although such an electron must tunnel through a barrier to escape, it is shown that the tunneling rate is not the rate-limiting step in electron loss; instead, it is the rate at which geometrical fluctuations cause the Coulomb potentials to exceed $5 \mathrm{eV}$ that determines the rate of electron loss. Because these rates are extremely slow, special techniques had to be introduced to allow results of dynamics simulations on more flexible models of $\mathrm{T}_{5}{ }^{3-}$ to be extrapolated to predict the behavior of the actual $\mathrm{T}_{5}{ }^{3-}$.
\end{abstract}

\section{Introduction}

A. Review of Experimental Findings on Negative Oligoucleotides. In recent gas-phase experiments ${ }^{1}$ on small multiply charged single-strand oligonucleotides, ${ }^{2}$ the Parks group has observed very slow (e.g., having rates of $10^{-4}$ to $1 \mathrm{~s}^{-1}$ ) sequential charge loss ${ }^{3}$ over temperatures ranging from 100 to $170{ }^{\circ} \mathrm{C}$. In these experiments, an oligonucleotide ion having a selected charge-to-mass ratio is retained in an ion trap at a given temperature and in an environment where collisions with reactive species or with walls are highly improbable. The abundance of the initial ion is monitored as time evolves, as are the abundances of other anions that are formed by electron loss. It is seen that the number of parent ions decays exponentially with time as daughter anions having one lower charge are formed. An example of the sequential decay of a fluores-

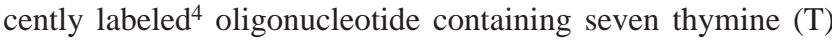
units that starts with a -4 charge state is shown in Figure 1. Here, the growth and decay of the -3 charge state are also shown as is the growth of the -2 charge state. The unimolecular rate constant for the -4 to -3 transition is $14 \times 10^{-3} \mathrm{~s}^{-1}$, and that for the -3 to -2 transition is $0.6 \times 10^{-3} \mathrm{~s}^{-1}$. Further decay of the -2 ion to generate an ion with charge -1 is not observed, at least on the time scales of the experiments.

The same kind of experiment has been carried out on oligonucleotides that contain no BODIPY fluorescent label and on species containing various bases (T, A, C, and $\mathrm{G}$ ) and base

* To whom correspondence should be addressed. E-mail: simons@ chemistry.utah.edu.

University of Utah.

$\doteqdot$ University of Gdansk.

$\S$ A holder of a Foundation for Polish Science (FNP) Award.

"Also Chemistry Department, University of Colorado, Denver, CO 80217.

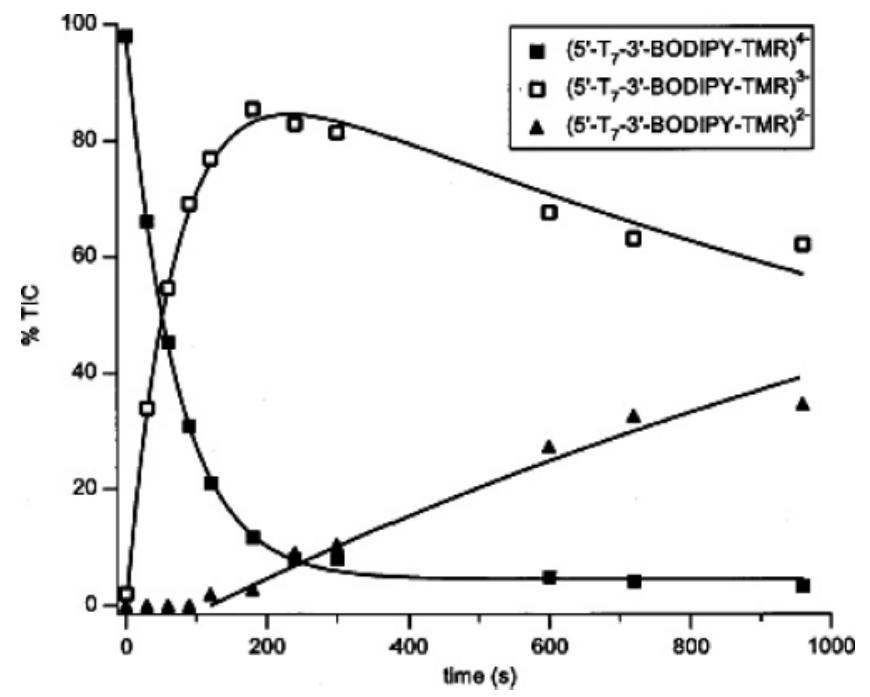

Figure 1. Percent abundances of $-4,-3$, and $-2 \mathrm{~T}_{7}{ }^{n-}$ ions as functions of time. Reprinted with permission from ref 1 . Copyright 2003 Elsevier.

combinations. In Figure 2 are shown the -3 to -2 rate constants for five such oligonucleotides containing a total of seven bases as functions of temperature, plotted in the conventional Arrhenius manner (i.e., $\ln k=\ln A-E^{\ddagger} / R T$ ).

The slopes of these Arrhenius plots suggest activation energies in the $22-24 \mathrm{kcal} \mathrm{mol}^{-1}$ range, and the intercepts correspond to preexponential $A$ factors ranging from $10^{11}$ to $10^{15} \mathrm{~s}^{-1}$. The following is the primary question we attempt to answer in the present paper: What is the physical process that causes the charge loss (electron ejection), and how is this process consistent with these activation energies and frequency factors? 


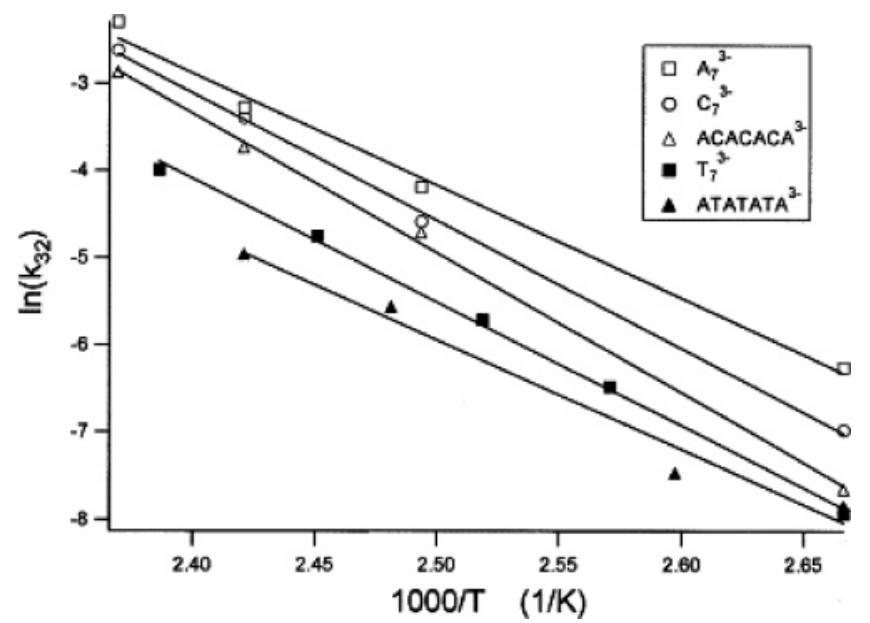

Figure 2. Arrhenius plots of the temperature dependences of the rate constants for -3 to -2 charge-loss transitions for a variety of oligonucleotides containing a total of seven bases.

A few observations about the rates of charge loss illustrated in Figures 1 and 2 are in order: (1) The rates for -4 to -3 charge loss are higher than those for -3 to -2 charge loss; in general, it is found that rates for $-n$ to $-(n-1)$ charge loss are higher than those for $-(n-1)$ to $-(n-2)$ charge loss. (2) The activation energies for a given $-n$ to $-(n-1)$ charge loss vary little as the base composition of the oligonucleotide changes, but the preexponential $A$ factors vary by several orders of magnitude. (3) All of the rates are very slow and correspond to charge losses taking place over seconds to hundreds of seconds.

It should be emphasized that the theoretical simulation of such processes is severely hindered by the extremely long time scales over which the electron-loss dynamics occurs. It is impossible to carry out straightforward molecular dynamics simulations (e.g., using time steps of $10^{-15} \mathrm{~s}$ ) of even a small oligonucleotide if one is required to follow the time evolution for durations of $1-1000 \mathrm{~s}$. Carrying out simulations for times in the $10^{-9} \mathrm{~s}$ range is within what is currently computationally feasible, so there are approximately 9 orders of magnitude in time that need to be dealt with. To overcome such long-timedynamics challenges, we introduce techniques that involve (a) monitoring the shorter-time dynamics of modified oligonucleotide systems whose intramolecular Coulomb repulsions (i.e., charge states) are (artificially) reduced to cause electron-loss events to occur on shorter time scales and with lower activation energy requirements and (b) then extrapolating results obtained on the artificial model systems to predict rates of electron ejection for the true oligonucleotide in its full charge state and with its full activation energy.

Because these techniques are not conventional, we will offer considerably more detail about them later in the methods section and when discussing our results, but first we need to explain how the internal Coulomb interactions among the phosphate groups both induce geometrical rigidity in the oligonucleotide and alter the strength with which the phosphate groups bind their electrons.

B. Roles of Repulsive Coulomb Interactions in Multiply Charged Anions. Before discussing how we carried out molecular dynamics simulations intended to simulate what may be going on in the Parks group's experiments, it is important to introduce one of the key concepts upon which we base these simulations. We know from our many studies of multiply charged anions that the intrinsic electron binding energy of an anion site can be modified, in a highly predictable manner, by

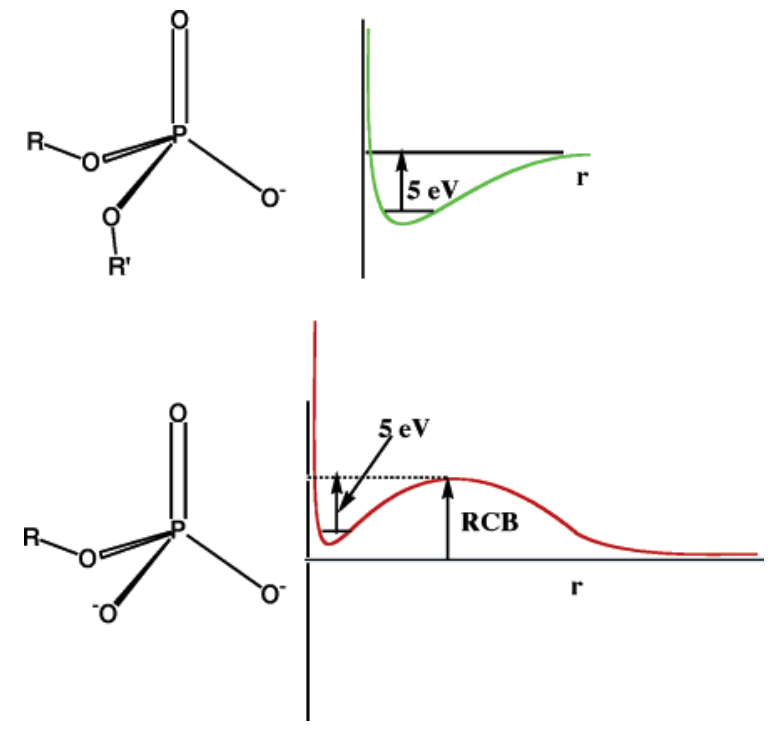

Figure 3. Electron binding potentials for $\mathrm{ROR}^{\prime} \mathrm{OPO}_{2}^{-}$(top) and $\mathrm{RPPO}_{3}{ }^{2-}$ (bottom).

the presence of nearby charged groups. For example, the electron binding energy of the $\mathrm{H}_{2} \mathrm{PO}_{4}{ }^{-}$anion, whose structure is shown in Figure 3, is $5 \mathrm{eV}$, but the doubly charged $\mathrm{HPO}_{4}{ }^{2-}$ anion (also shown in Figure 3) is actually unstable with respect to electron detachment by $0.3 \mathrm{eV}$.

To understand the origin of this very large change in electronic stability in moving from $\mathrm{H}_{2} \mathrm{PO}_{4}{ }^{-}$to $\mathrm{HPO}_{4}{ }^{2-}$, one need only consider the potentials that bind an electron to the underlying molecular framework for each of these species. For $\mathrm{H}_{2} \mathrm{PO}_{4}{ }^{-}$, this potential is centered on one of the oxygen atoms and has the radial form shown in the top half of Figure 3. This strongly attractive potential has a depth that produces a bound state $5 \mathrm{eV}$ below $\mathrm{H}_{2} \mathrm{PO}_{4}+\mathrm{e}^{-}$. This potential reflects what we call the "intrinsic" electron binding energy of this oxygen site in the phosphate group. In contrast, the potential associated with the $\mathrm{HPO}_{4}{ }^{2-}$ dianion has two contributions-one attractive part exactly like the $5 \mathrm{eV}$ deep potential of $\mathrm{H}_{2} \mathrm{PO}_{4}{ }^{-}$and a second purely repulsive part that reflects the Coulomb repulsion caused by the second negative site in this dianion. The combination of these two parts produces a total potential of the form shown in the bottom portion of Figure 3 .

It is important to see that the Coulomb repulsion exerted by the second negative site has two effects: (a) It shifts the energy of the (previously bound) anion state upward by an amount that can be estimated in terms of the distance $R$ between the two negative sites in the dianion: $e^{2} / R=(14.4 \mathrm{eV}) /[R(\AA)]$. (b) It produces a so-called repulsive Coulomb barrier $(\mathrm{RCB})$ whose height is exactly this same $e^{2} / R$.

For the $\mathrm{HPO}_{4}{ }^{2-}$ case under discussion, this means that although the dianion has an energy $0.3 \mathrm{eV}$ above zero (i.e., higher than $\mathrm{HPO}_{4}^{-}+\mathrm{e}^{-}$), to undergo autodetachment, the electron must tunnel through a barrier whose height is RCB. In an earlier publication, we estimated ${ }^{5}$ the rate of tunneling to be ca. $10 \mathrm{~s}^{-1}$ for this particular dianion.

\section{Proposed Roles of Internal Coulomb Repulsions in Oligonucleotides}

A. Coulomb Interactions Fluctuate as Dynamical Motions Cause Interion Distances To Fluctuate. It would seem that the singly charged phosphate units (note, as explained earlier, some of the phosphate units in the oligonucleotides are neutral) in the experiments should behave similar to the $\mathrm{H}_{2} \mathrm{PO}_{4}{ }^{-}$anion 

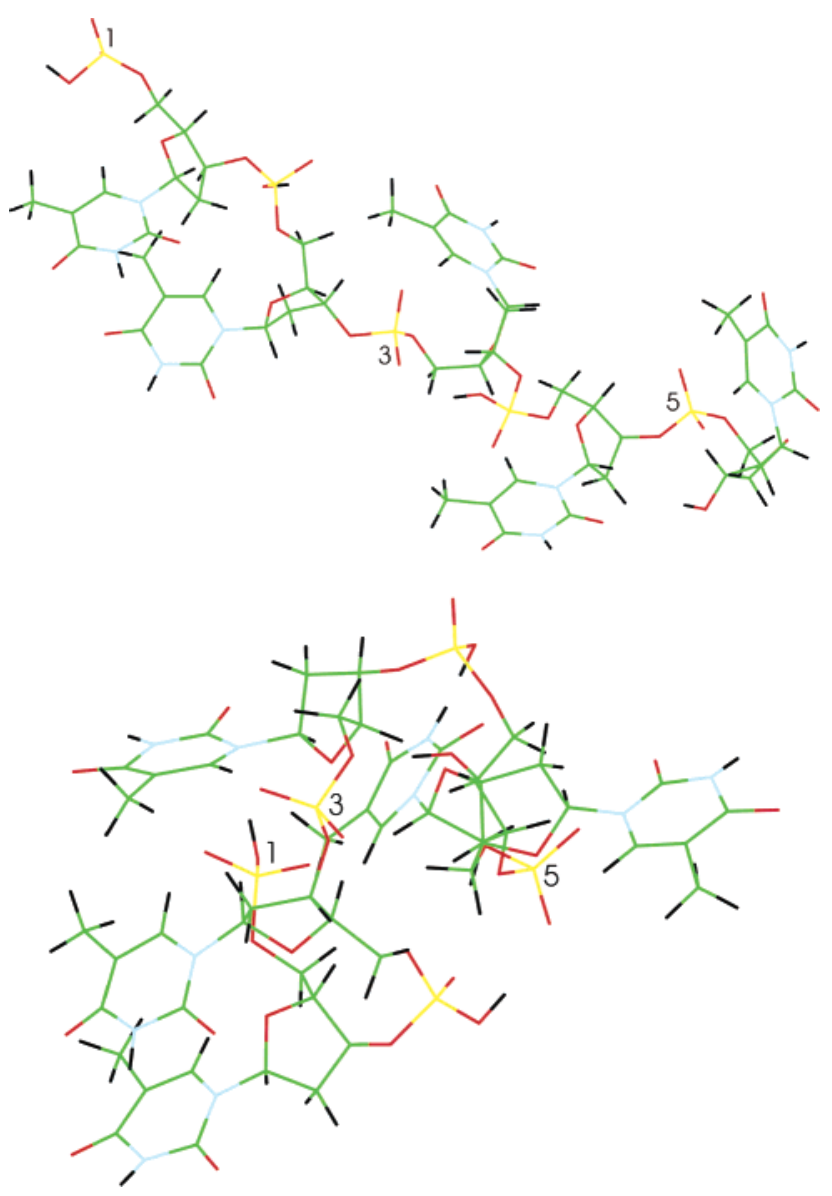

Figure 4. Examples from molecular dynamics simulation of (a, top) an extended structure of $\mathrm{T}_{5}{ }^{3-}$ and (b, bottom) a more compact structure visited less frequently during dynamical motions.

discussed above and thus bind their electrons by ca. $5 \mathrm{eV}$. However, each of the negative phosphate units in the oligonucleotide is in reasonable proximity to the other negative phosphates. As a result, each of these anion sites will have its intrinsic electron binding energy $(5 \mathrm{eV})$ reduced by an amount that depends on the distances $R_{I, J}$ to the other negative sites. Specifically, the "instantaneous" electron binding energy of site $I$ will be $5 \mathrm{eV}-\Sigma_{J} e^{2} / R_{I, J}=5 \mathrm{eV}-\Sigma_{J}(14.4 \mathrm{eV}) / R_{I, J}$. So, any site whose Coulomb repulsion energy exceeds $5 \mathrm{eV}$ can be subject to autodetachment.

Let us consider in more detail the nature of the intramolecular Coulomb repulsions that arise in the kind of oligonucleotides examined in the experiments by considering one such species containing five thymine units and three negatively charged phosphates, $\mathrm{T}_{5}{ }^{-3}$. Because of the Coulomb repulsions among the three negative charges, the most probable structures for such a species are expected to be rather extended as shown in Figure 4a. This, of course, does not mean that dynamical fluctuations will not allow the structure to become more compact, but certainly in the gas phase where solvation influences are absent, such extended structures are more often visited than are much more compact structures. The particular structure in Figure 4a came from a molecular dynamics simulation carried out at $T=$ $170{ }^{\circ} \mathrm{C}$ and is representative of the kinds of structures this anion frequently accesses during its dynamical movements. From this structure we can see that the three negative phosphate sites (labeled 1, 3, and 5) are indeed arranged in a quasi-linear extended geometry as the distances among the 1,3 , and 5 phosphate sites, $r_{1,3}=11.6 \AA, r_{3,5}=11.1 \AA$, and $r_{1,5}=21.3 \AA$, suggest. Of course, dynamical fluctuations cause the $\mathrm{T}_{5}{ }^{3-}$

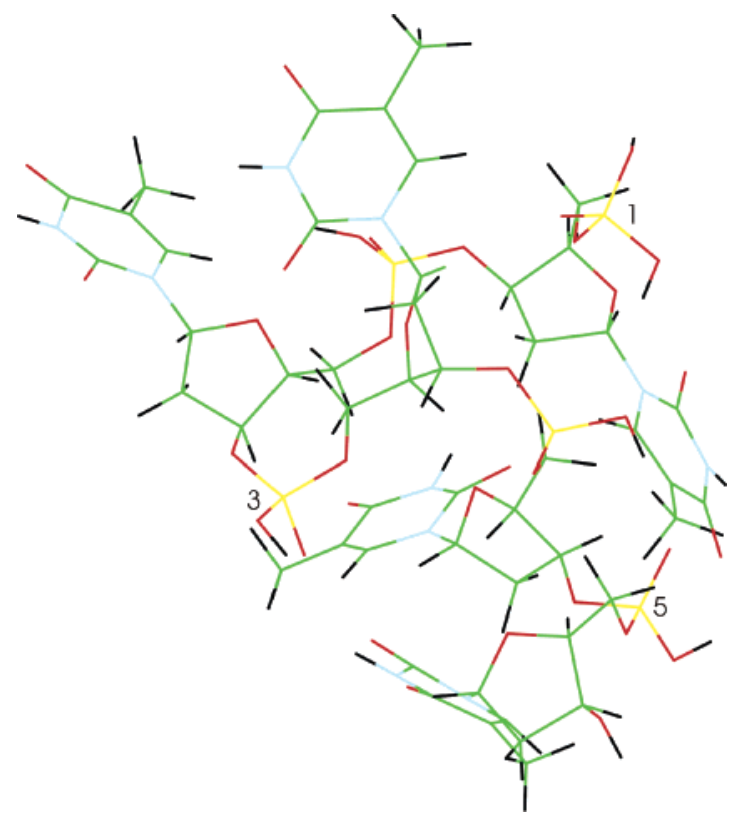

Figure 5. Example from molecular dynamics simulation of the highly probable more compact structure of $\mathrm{T}_{5}{ }^{3-}$ resulting from turning off the charges on the three negative phosphate groups.

structure to (infrequently) access more compact structures such as we show in Figure $4 \mathrm{~b}$, where $r_{1,3}=5.63 \AA, r_{3,5}=6.31 \AA$, and $r_{1,5}=8.04 \AA$.

It is also useful to consider what the highly probable structures of $\mathrm{T}_{5}{ }^{3-}$ would look like if the Coulomb interactions among the three negative sites were absent. We also carried out $T=170$ ${ }^{\circ} \mathrm{C}$ molecular dynamics simulations of gas-phase $\mathrm{T}_{5}{ }^{3-}$ in which we simply turned off the -1 charges on phosphate groups 1,3 , and 5 , and we obtained highly probable structures similar to that shown in Figure 5.

It is clear that this structure is more compact than the highly probable extended structure shown earlier for the -3 charged $\mathrm{T}_{5}{ }^{3-}$. In fact, the distances among the 1,3 , and 5 phosphate groups in this compact structure are $r_{1,3}=8.76 \AA$, $r_{3,5}=5.80$ $\AA$, and $r_{1,5}=11.7 \AA$, which are considerably shorter than those seen in Figure 4a, although they are comparable to the distances observed in the infrequently observed structure of -3 charged $\mathrm{T}_{5}{ }^{3-}$ shown in Figure 4b.

With these geometry ideas in mind, let us now consider the potential experienced by an electron on one of the negative phosphate units in the true -3 charged $\mathrm{T}_{5}{ }^{3-}$. First, each of the phosphates has an intrinsically attractive potential deep enough to produce a state bound by $5 \mathrm{eV}$. However, each phosphate also has a repulsive Coulomb potential that is induced by the presence of the other two negative phosphate sites. We note that this repulsive potential is not the same for the first, third, and fifth phosphate units. In particular, the central negative phosphate (unit 3) has a higher Coulomb potential because $\Sigma_{J^{-}}$ $(14.4 \mathrm{eV}) / R_{I, J}$ is larger for this site than for the two terminal sites. For example, in the $\mathrm{T}_{5}{ }^{3-}$ structure shown in Figure $4 \mathrm{~b}$, the Coulomb energies at the three sites are $V(3)=(14.4 / 5.63$ $+14.4 / 6.31) \mathrm{eV}=4.84 \mathrm{eV}, V(1)=(14.4 / 5.63+14.4 / 8.04)=$ $4.35 \mathrm{eV}$, and $V(5)=(14.4 / 6.31+14.4 / 8.04)=4.07 \mathrm{eV}$. For the more compact structure shown in Figure 5, the potential at the central phosphate is $V(3)=(14.4 / 8.76+14.4 / 5.80)=4.13$ $\mathrm{eV}$. Of course, these values are the Coulomb energies when the three phosphate sites are located in the positions depicted in the figures. Only when dynamical fluctuations cause the sites to approach more closely will the Coulomb energies reach the 


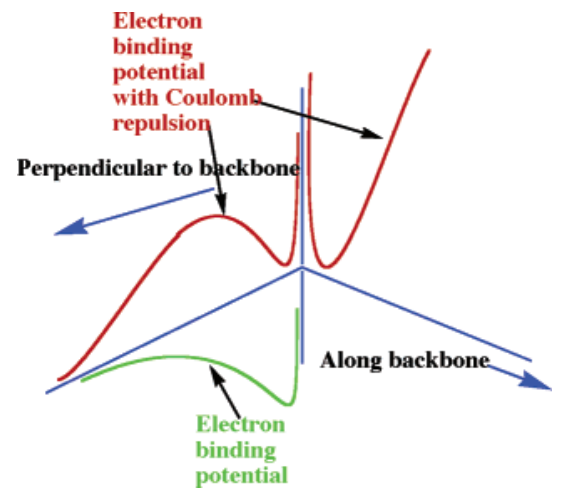

Figure 6. Depiction of the $5 \mathrm{eV}$ intrinsic binding potential (green) as well as the total electron binding potential (red) as functions of the electron-backbone distance along directions parallel to and perpendicular to the $\mathrm{T}_{5}{ }^{3-}$ backbone.

$5 \mathrm{eV}$ critical value needed to render the phosphate's electron capable of detaching.

For every instantaneous geometry of the $\mathrm{T}_{5}{ }^{3-}$ ion, each of its phosphate sites has a total potential that displays an electronbackbone distance dependence of the form shown in Figure 6.

Here, we denote in green the intrinsically attractive potential that would bind the electron by $5 \mathrm{eV}$ if no Coulomb repulsions existed, while in red we show the total (intrinsic plus repulsive Coulomb) potential. Note that the total potential becomes more and more repulsive as one moves in directions along the $\mathrm{T}_{5}{ }^{3-}$ backbone (i.e., toward either of the other negative phosphates) but has the form of a barrier and subsequent decay in directions perpendicular to the backbone. It is the latter directions that allow the electron to escape by autodetaching and thus generating charge loss.

Let us now bring these concepts of Coulomb interactions and electron-molecule potentials together in a way that defines how we think about the electron ejection process going on in the Parks experiments. The fundamental assumptions of the molecular dynamics calculations we used to simulate these experiments are that (a) dymamical motions of the oligonucleotide (e.g., $\mathrm{T}_{5}{ }^{3-}$ for the case examined in this paper) cause the distances $R_{I<J}$ among the negatively charged phosphate units to fluctuate, (b) as the $R_{I, J}$ distances change, so do the Coulomb destabilizations $\Sigma_{J}(14.4 \mathrm{eV}) /\left[R_{I, J}(\AA)\right]$ associated with each phosphate site, (c) whenever the total Coulomb destabilization at a site exceeds $5 \mathrm{eV}$, that site is susceptible to electron detachment, and (d) the rate at which geometrical fluctuations cause the Coulomb destabilization at a site to exceed $5 \mathrm{eV}$ will determine the rate of electron detachment from that site.

An example of the progression in the binding potential associated with a particular phosphate group at three times within a typical dynamical movement is illustrated in Figure 7.

At time 1, the electron cannot detach because the total potential binds it. However, at times 2 and 3, the electron can detach, but it must tunnel through the RCB on the corresponding energy surface to escape. Such tunneling is more facile at time 3 than at time 2 for reasons that we discuss below. In fact, it is important to analyze this tunneling rate so we can determine whether tunneling will be the rate-limiting step for electron loss. As we hinted earlier, it turns out that tunneling is generally not the slow step for charge loss; instead, it is the rate at which structural fluctuations cause fluctuations in the electronbackbone potential to destabilize the $5 \mathrm{eV}$ bound anion state that is rate-limiting. Let us now see why this is the case.

B. Even When Coulomb Repulsions Exceed 5 eV, a Barrier Must Be Tunneled Through. Thus far in describing

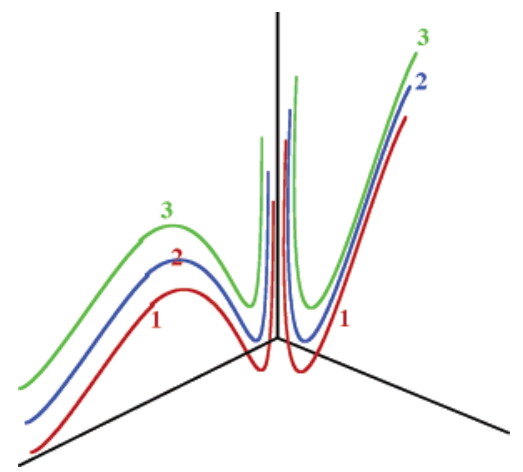

Figure 7. Total electron binding potential at three times along and perpendicular to the $\mathrm{T}_{5}{ }^{3-}$ backbone.

the model put forth here, we suggest that fluctuations in the Coulomb potential at a given site $(I)$ will cause the $5 \mathrm{eV}$ bound electron to become destabilized to an extent that permits the electron to autodetach. However, as just noted, we need to consider whether it is the rate at which the local Coulomb potentials exceed $5 \mathrm{eV}$ or the rate at which the metastable state tunnels through the barrier that ultimately determines the rate of charge loss. To this end, we recall that, in an earlier effort, ${ }^{5}$ we estimated the rate at which an electron of energy $E=\mathrm{RCB}$ $-5 \mathrm{eV}$ tunnels through a barrier of the form $e^{2} / r$ having an energy maximum equal to RCB. We ultimately expressed the rate as $\sim 10^{15} \exp \left\{-\left(2(\mathrm{RCB}-E)^{1 / 2}\right)(1 / E-1 / \mathrm{RCB})\right\}$. The factor of $10^{15}$ represents the frequency $\left(\mathrm{s}^{-1}\right)$ at which the electron undergoes motion within the phosphate's oxygen orbital (i.e., this is the Bohr orbit frequency).

Using $\mathrm{RCB}=5 \mathrm{eV}+\delta$, we obtain rates of $10^{2}, 10^{8}$, and $10^{10} \mathrm{~s}^{-1}$ for $\delta=0.1,0.2$, and $0.3 \mathrm{eV}$, respectively. This means that if the total Coulomb energy at a phosphate site exceeds the intrinsic $5 \mathrm{eV}$ binding energy by as little as $0.1 \mathrm{eV}$, the rate of tunneling through the RCB will exceed the rates at which Parks observes charge loss. Thus, tunneling will not be the ratelimiting step in the charge-loss process except for a very small population of ions whose Coulomb energies lie very close to 5 $\mathrm{eV}$. Instead, we suggest it is the rate at which the total Coulomb energy at the various phosphate sites reaches (and slightly exceeds $5 \mathrm{eV}$ ) that likely is the rate-limiting step.

\section{Methods for Carrying Out the Molecular Dynamics Simulations}

Because the charge-loss events observed in the Parks experiments take place on time scales of $1-1000 \mathrm{~s}$, it is foolish to attempt to simulate them by carrying out straightforward molecular dynamics calculations. Such computations typically use time steps of $10^{-15} \mathrm{~s}$, so a single trajectory lasting $1 \mathrm{~s}$ would require $10^{15}$ propagations of all the atomic positions and momenta, each step of which would involve a few hundred floating point calculations. Even with 1000 computers used in parallel and having speeds in the $5 \times 10^{9} \mathrm{~Hz}$ range, such a $1-1000 \mathrm{~s}$ trajectory would not be feasible. Therefore, we need to use special "tricks" to estimate the long-time $(1-1000 \mathrm{~s})$ behavior of the $\mathrm{T}_{5}{ }^{3-}$ system of interest. In particular, we employed the following two devices to allow us to achieve our goals.

(a) We carried out dynamical simulations on a series of modified $\mathrm{T}_{5}{ }^{3-}$ anions in which the charges on each of the three negative phosphate units were scaled by an amount $q$ ranging from 0.3 to 0.9 in steps of 0.1 . The idea behind this chargescaling device is that the species with $q<1.0$ will be less rigid than the true $\mathrm{T}_{5}{ }^{3-}$ and thus more prone to allow their phosphate 
groups to approach one another closely enough to make their inter-phosphate Coulomb potentials large. Note that although we utilize the reduced charges within the dynamics simulations (i.e., to determine the anion's movements), we still assume each phosphate carries a full -1 charge when evaluating the Coulomb potential at each phosphate site. Then, by examining the results of our dynamics simulations (e.g., the frequencies with which Coulomb potentials at the phosphate sites exceed a specified threshold $V$ ), we are able to extrapolate our findings to $q=1.0$ and to thus represent the behavior of the true $\mathrm{T}_{5}{ }^{3-}$. This particular trick allows us to use data collected on the more compact and flexible $(q<1.0)$ systems, where we observe large numbers of "events" (i.e., when the Coulomb potential exceeds the threshold energy $V$ ) and thus have low statistical error, to estimate the event frequency for the $q=1.0$ system of interest.

(b) We analyzed the frequencies at which the Coulomb destabilization at a given negative phosphate site exceeded a specified energy $V$ ranging from 4.2 to $4.5 \mathrm{eV}$ in steps of 0.1 $\mathrm{eV}$. Within this range of $V$ values, we observed substantial numbers of events, so we could evaluate the rate of occurrence of such events with good statistical significance. By then introducing a model for how the rates at which events vary over the 4.2-4.5 eV range, we were able to extrapolate to higher $V$ values and thus predict the rates at which the Coulomb energies would exceed 5 or $5.1 \mathrm{eV}$ in $\mathrm{T}_{5}{ }^{3-}$.

These extrapolation devices were essential because, as we earlier stated, if we were to simply carry out dynamics simulations on the $q=1.0 \mathrm{~T}_{5}{ }^{3-}$ system and monitor the frequency with which the Coulomb potential at a phosphate site exceeded $5 \mathrm{eV}$, we would fail because, as the Parks experiments show, this frequency is ca. $1 \mathrm{~s}^{-1}$ or less. It therefore was crucial to introduce methods that allow us to follow the shorter-time dynamics for less rigid (i.e., $q<1.0$ ) model systems and to monitor the frequencies of attaining smaller (i.e., $V \leq 4.5 \mathrm{eV}$ ) Coulomb potentials while then extrapolating to $q=1.0$ and to higher $V$ values.

Before leaving this discussion, it is important to emphasize that requiring fluctuations to produce Coulomb potentials at a phosphate site equal to $5 \mathrm{eV}$ is not equivalent to overcoming an activation barrier of $5 \mathrm{eV}$. As Figures 4 and 5 show, the primary backbone structure of the $\mathrm{T}_{5}{ }^{3-}$ ion constrains the nearneighbor inter-phosphate distances to be in the 5-8 $\AA$ range (i.e., to move more than ca. $8 \AA$ away, one would have to break covalent bonds). As such, the Coulomb potentials at, for example, site 3 are in the $4 \mathrm{eV}$ range even at the minimumenergy structure and in the absence of any thermal excitation that might induce shortening in the inter-phosphate distances. Hence, by no means does it require that $5 \mathrm{eV}$ of kinetic energy be deposited into one or more of the inter-phosphate repulsive potentials to achieve the conditions for electron loss. It is only necessary to move from the Coulomb potential at the minimumenergy structure of $\mathrm{T}_{5}{ }^{3-}$ to a structure for which at least one phosphate site has a $5 \mathrm{eV}$ Coulomb potential.

The MD simulations were carried out using Amber 7.0 with the parm99 force field. ${ }^{6}$ The HF/6-31G/RESP methodology ${ }^{7}$ was used to derive charges for the DNA fragment. The starting structure was first subjected to short minimization to remove bad steric contacts. Simulations were carried out in vacuo at constant temperature (443 K) using the weak-coupling Berendsen method with a relaxation time of 1.0 ps. Data for 1000 ps runs were collected for all atoms in the system every 500 steps corresponding to $1 \mathrm{ps}$.

As an initial structure for $\mathrm{T}_{5}{ }^{3-}$ the extended geometry of five $\mathrm{T}$ units was used (each of which taken from LEaP) ${ }^{6}$ and was then thermalized for $50 \mathrm{ps}$. The dynamics calculations used a time step of $10^{-15} \mathrm{~s}$ and involved probing the molecular geometry and its internal Coulomb energy every 1 ps for a total of 7000 ps. Statistics were obtained every 1000 ps to check for variations within the full 7000 ps trajectory.

\section{Results}

A. Distributions in Coulomb Potentials for Various $q$ Values. For each 1000 ps segment of our molecular dynamics simulation of the various $\mathrm{T}_{5}{ }^{3-}$ species whose negative phosphate charges are scaled by $q$, we collected (at 1 ps intervals) information about the Coulomb potentials at the three phosphate sites. We represent these data in histogram form as shown in Figure 8, where we plot the number of events in which a given value of the Coulomb potential is realized for each site. We show these data for $q=0.4,0.7$, and 0.9 (we also obtained data for $q=0.3,0.5$, and 0.6 ), and we show histograms from more than one $1000 \mathrm{ps}$ time slice for each $q$ to give the reader some idea of the statistical fluctuations realized as time evolves.

Four observations are important to make about these data: (1) The central phosphate site (shown in red) has the highest Coulomb potential (for reasons that we explained earlier). (2) This central site has, for $q=0.9$, a high occurrence of potentials in the $4.2 \mathrm{eV}$ range. Thus, structural fluctuations need input only enough energy to cause this site to have a Coulomb energy of ca. $5 \mathrm{eV}$ to become unstable with respect to electron loss and able to promptly tunnel through the Coulomb barrier. (3) For small $q$, the number of events corresponding to a phosphate site having a large Coulomb potential is larger than the number of such events for larger $q$. This, of course, is because the small- $q$ species' dynamics allows the phosphate units to approach more closely and thus experience high Coulomb interactions more often. (4) The fluctuations from one $1000 \mathrm{ps}$ sample to another are small. This gives us good confidence in the statistical significance of our data.

Another observation that is important to extract from these plots is the very low probability of observing a Coulomb potential in the 5.0-5.1 eV range. For the more flexible $q=$ 0.4 species, the distribution of Coulomb potentials at the central phosphate site suggests that $V=5.0 \mathrm{eV}$ can be realized albeit quite infrequently. However, for the more rigid $q=0.9$ data, $V$ $=5.0 \mathrm{eV}$ shows essentially zero probability. This illustrates the challenge we face in attempting to estimate the frequency with which $V=5.0 \mathrm{eV}$ or $V=5.1 \mathrm{eV}$ can be experienced in the real $(q=1.0) \mathrm{T}_{5}{ }^{3-}$. Recall that our strategy will be to extrapolate our low- $q$ data for $V=4.2-4.5 \mathrm{eV}$ (where we experience many events and thus have good numerical significance) to $q=1.0$ and to $V=5.0-5.1 \mathrm{eV}$ to gain an estimate of the rate at which real $\mathrm{T}_{5}{ }^{3-}$ accesses Coulomb potentials in the 5.0-5.1 eV range.

B. Rates at Which Various Potentials $\boldsymbol{V}$ Are Realized. At every 1 ps interval during each 1000 ps time slice of the dynamical simulation for a given value of $q$, we monitor the Coulomb potential at each of the three phosphate units. Whenever this potential exceeds the value $V$, we note the time at which this occurs. We subsequently monitor the potential at this site to determine when the potential again exceeds the value $V$, and we note when this occurs. As a result, we are able to collect data about the time intervals between events (i.e., exceeding some specified potential $V$ ) at each of the three phosphate sites. By analyzing the distribution (which we observe to be well represented as exponential) of delay times between events at a given site, we can compute the average delay time as well as the average number of events per unit time. It is 

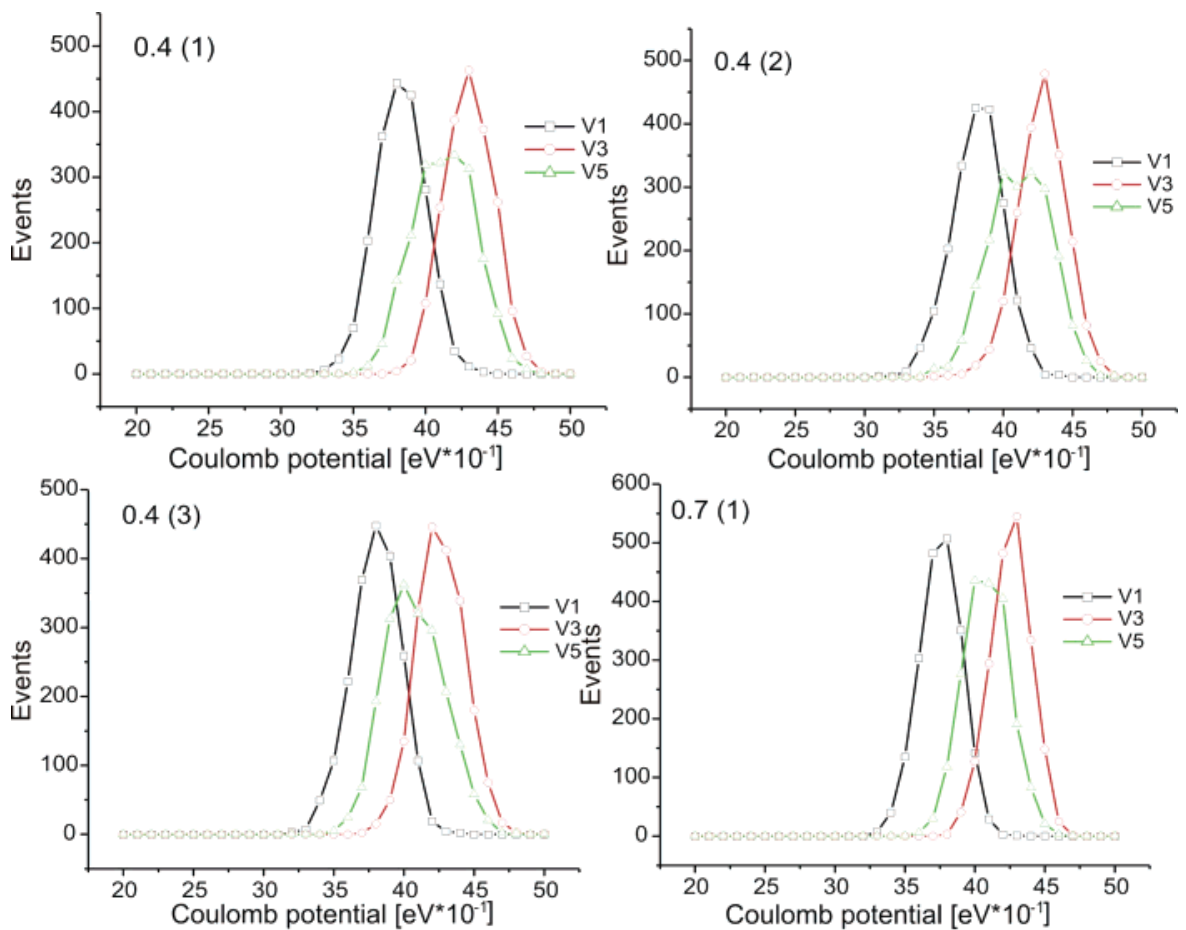

$0.7(1)$
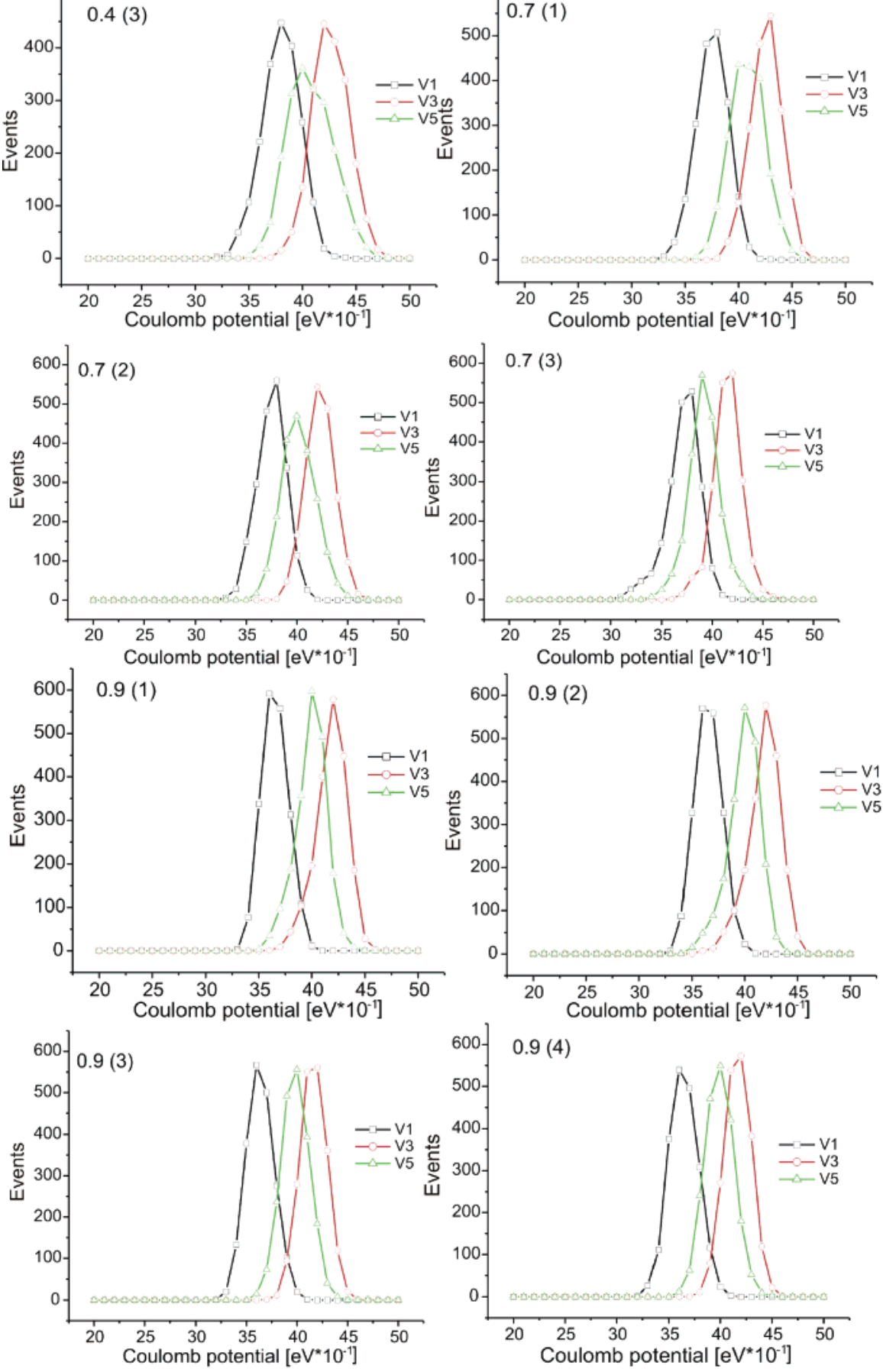

Figure 8. Histograms showing the number of times any of the three negative phosphate sites realize a given Coulomb energy (eV). Each histogram results from a $1000 \mathrm{ps}$ time slice that is examined every 1 ps. For $q=0.4$ and 0.7 , histograms from three 1000 ps time slices are shown; for $q=$ 0.9 , four such histograms are shown. 

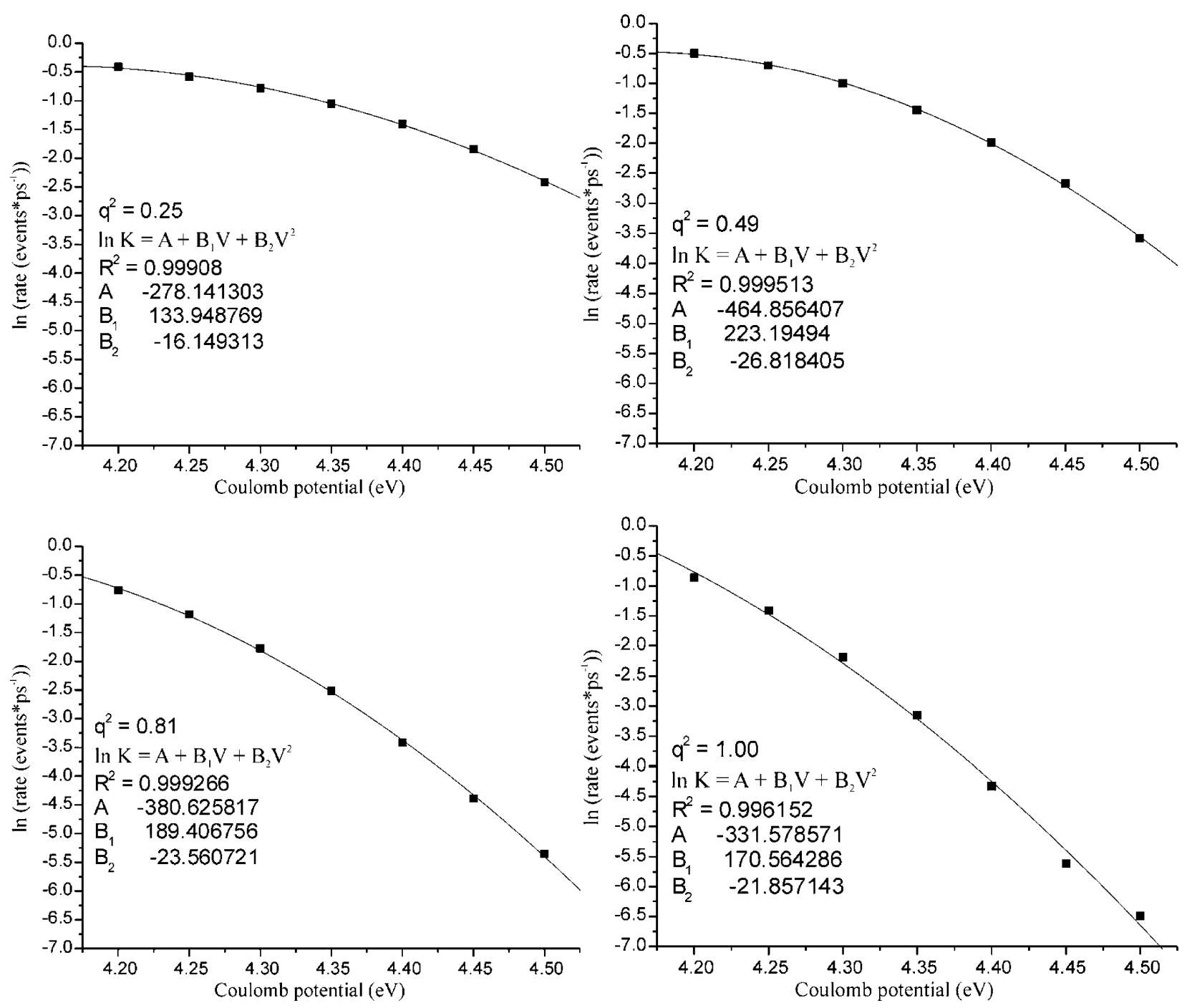

Figure 9. Natural logarithm of the number of events per picosecond vs the Coulomb potential at the central site (3) for $q=0.5,0.7,0.9$, and 1.0 .

such a rate of events per unit time that we use to suggest the rate at which electron detachment will take place.

In Figure 9 we show results of such an analysis of how often specified values of the Coulomb potential are realized. We illustrate the data for $q=0.5,0.7$, and 0.9 and for values of the Coulomb potential at which our simulations experience enough events to be statistically significant.

To obtain the rate data shown in Figure 9 for $q=1.0$, we could not use results from our molecular dynamics simulations on the true (i.e., $q=1.0$ ) $\mathrm{T}_{5}{ }^{3-}$ because the number of events realized for $V=4.5-4.3 \mathrm{eV}$ was very small (i.e., and thus may not be numerically accurate). We therefore used our data for smaller $q$ values to extrapolate to $q=1.0$ and thus gain a better description of the true $\mathrm{T}_{5}{ }^{3-}$ rates. In Figure 10 we show our rate data plotted for a given value of the Coulomb potential as $q$ is varied.

We plot these data vs $q^{2}$ because the internal Coulomb potentials among the three negative phosphate sites scale quadratically with $q$. From each such graph we were then able to extrapolate to $q=1.0$ to gain our estimate of the rates appropriate to $\mathrm{T}_{5}{ }^{3-}$, and it is these rate data that appear in Figure 9 under the $q^{2}=1.0$ label. Note that although the $R^{2}$ residuals for the plots of $\ln$ (rate) vs $q^{2}$ are only modest, our extrapolated rates can be good because the range of $q^{2}$ over which we need to extrapolate is very small.

Before closing this section, we want to comment on an approach that we have not examined, that of using statistical theories such as RRKM theory to estimate the rate of electron loss. In such an approach, one must first identify a transition state (TS) at which one subsequently computes the appropriate density of states and the integrated number of states. However, in the charge-loss process studied here, there is no single barrier on the energy surface that connects reactants and products. Instead, any combination of the locations of the three negative phosphate sites $(1,3$, and 5$)$ that causes the Coulomb energy at site 3 to exceed $5.1 \mathrm{eV}$ can give rise to charge loss. Hence, there are a multitude of reaction paths connecting reactants and products, so the straightforward application of RRKM-like theories is complicated in such cases, and we are still struggling with how to properly carry out this kind of calculation.

C. Extrapolation to $\boldsymbol{V}=\mathbf{5} \mathrm{eV}$ or $\boldsymbol{V}=\mathbf{5 . 1} \mathrm{eV}$. Given the rates (in events per picosecond) at which various values $(V)$ of the Coulomb potential at the central phosphate site are realized for the -3 charged $\mathrm{T}_{5}{ }^{3-}$ anion, we need to extrapolate our rate data for $V=4.2-4.5 \mathrm{eV}$, where the number of events in our 7 ns simulations was large enough to be statistically significant, to the values $V=5.0 \mathrm{eV}$ and $V=5.1 \mathrm{eV}$. Recall that $5 \mathrm{eV}$ is thought to be the minimum value for the Coulomb potential to render the phosphate site unstable to electron detachment, and $5.1 \mathrm{eV}$ is the value that also allows the rate of tunneling through the repulsive Coulomb barrier to exceed ca. $100 \mathrm{~s}^{-1}$.

To effect the desired extrapolation, we need to decide on a reasonable functional form for how the rate should vary with $V$. Because our model assumes that dynamical fluctuations in 

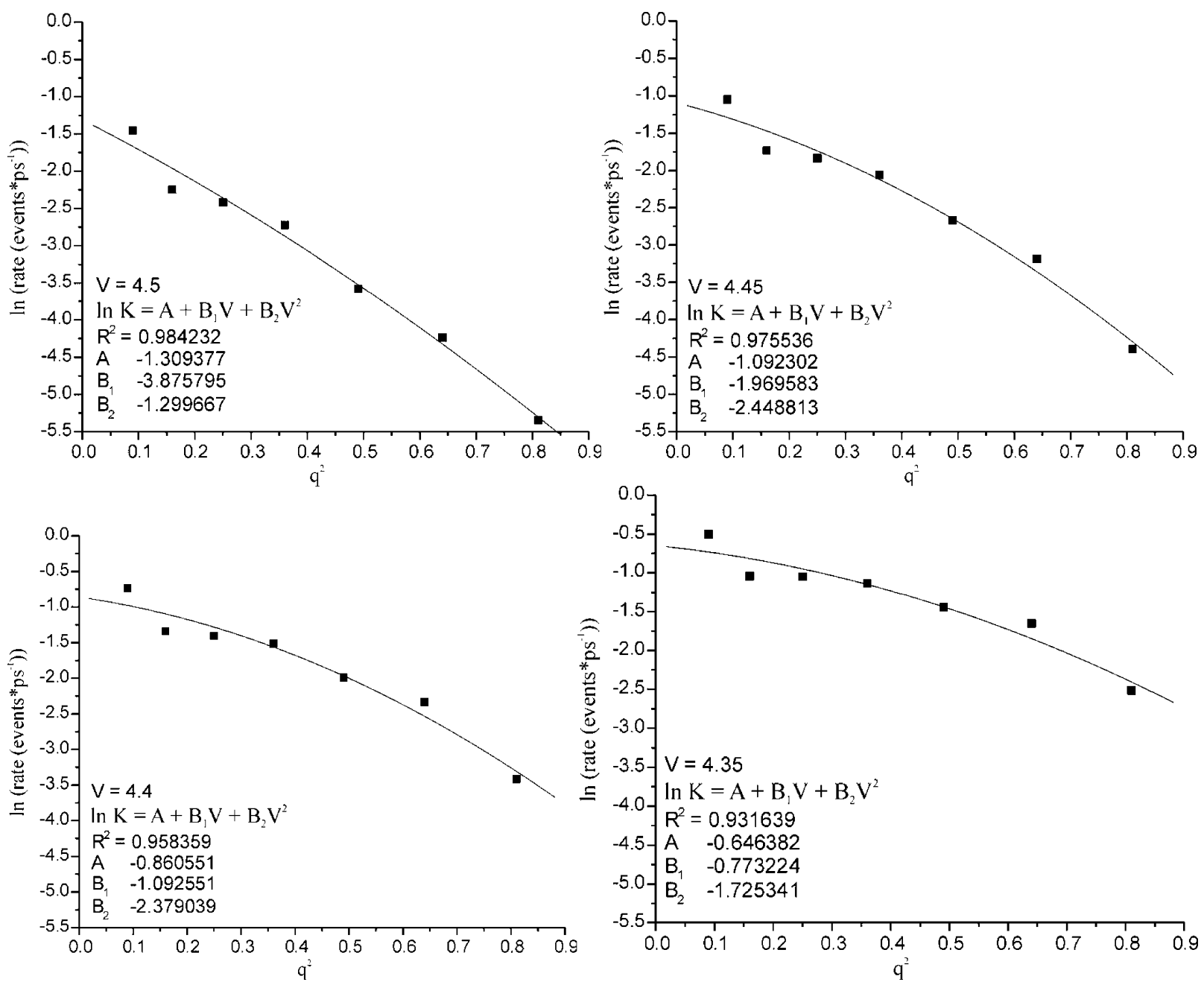

Figure 10. Natural logarithm of rates (events per picosecond) vs $q^{2}$ for various values of the Coulomb potential.

the inter-phosphate distances are responsible for fluctuations in the Coulomb potentials $(V)$, and because such structural changes should follow canonical Boltzmann statistics, it seems reasonable to assume that the probability of accessing a Coulomb repulsion energy $V$ should decay exponentially with $V$. However, recall that the energy $E$ required to cause the Coulomb potential to increase from its value of ca. $4.2 \mathrm{eV}$ at the minimum-energy structure of $\mathrm{T}_{5}{ }^{3-}$ to 5 or $5.1 \mathrm{eV}$ is considerably less than $5 \mathrm{eV}$ (i.e., is expected to be in the 22$23 \mathrm{kcal} \mathrm{mol}^{-1}$ range) and more like $0.8-0.9 \mathrm{eV}$. Nevertheless, we expect the activation energy $E$ to be monotonically related to $V$. For these reasons, we decided to express the frequency of event occurrences in the Arrhenius form, rate $=A \exp (-E /$ $R T$ ), where $T$ determines the average internal energy of the $\mathrm{T}_{5}{ }^{3-}$ ions in the Parks experiments carried out at this temperature, and to assume that $E$ depends monotonically on $V$. This model assumes that bringing any phosphate site into a state where its Coulomb potential renders it unstable to electron loss (i.e., $V$ $=5.0-5.1 \mathrm{eV}$ ) is a process that can be characterized by a frequency factor, $A$ (how often the phosphate site is buffeted by other nearby negative sites), and a probability $(\exp (-E / R T))$ that such buffeting has enough energy to raise the Coulomb potential to $V$. Assuming that $E$ is directly related to $V$, we therefore expect that a plot of $\ln ($ rate) vs $V$ should be of use in effecting the extrapolation to larger $V$ values.

Of course, we have already shown such plots in Figure 9, but we repeat the specific $q=1.0$ plot in Figure 11, where we plot $\ln$ (rate) vs the magnitude of the Coulomb potential $V$ in

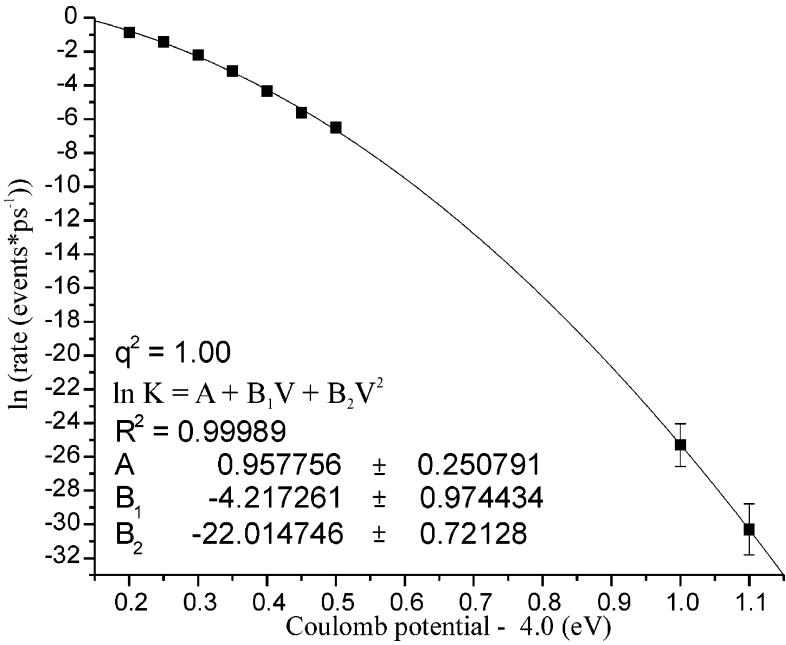

Figure 11. Natural logarithm of the rate (events per picosecond) vs the Coulomb potential in excess of $4.0 \mathrm{eV}$. Also shown on the right are the rates extrapolated to 5.0 and $5.1 \mathrm{eV}$.

excess of $4.0 \mathrm{eV}$. We also show the result of least-squares fitting these rate data to a polynomial of second order in $V$.

The seven data points at the top of this figure represent the rate data we obtained for the $\mathrm{T}_{5}{ }^{3-}$ system (by extrapolating our modified $\mathrm{T}_{5}{ }^{3-} q=0.3$ to $q=0.9$ rate data to $q=1.0$ ) assuming values of $V=4.2-4.5 \mathrm{eV}$ to be the "critical" values of the Coulomb potential. These seven data points were then fit to a functional form, $\ln ($ rate $)=A+B_{1}(V-4.0)+B_{2}(V-4.0)^{2}$, 


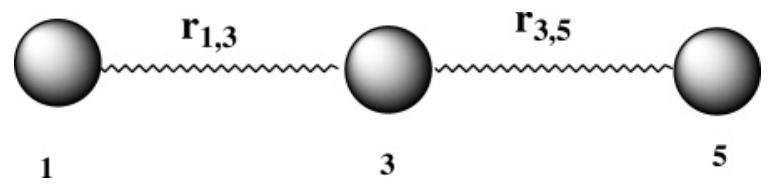

Figure 12. Model of three negative phosphate sites $(1,3,5)$ connected by harmonic potentials.

and extrapolated to $V=5.0 \mathrm{eV}$ and to $V=5.1 \mathrm{eV}$. The error bars shown on the $V=5.0$ and $5.1 \mathrm{eV}$ data reflect the errors arising in the $A, B_{1}$, and $B_{2}$ coefficients.

It can be seen that the $R^{2}$ residual of the fit is excellent and the uncertainties in the fitting parameters are small. As a result, the uncertainties in the extrapolated values of $\ln$ (rate) are not very large. In particular, the extrapolated values allow us to estimate the rate of accessing $V=5.0 \mathrm{eV}$ to be $(0.3-3.8) \times$ $10^{-11} \mathrm{ps}^{-1}$ or $3-38 \mathrm{~s}^{-1}$, and of accessing $V=5.1 \mathrm{eV}$ to be $(2-46) \times 10^{-14} \mathrm{ps}^{-1}$ or $0.02-0.5 \mathrm{~s}^{-1}$.

Clearly, the time scales we estimate for accessing the $5.1 \mathrm{eV}$ threshold needed to render the central phosphate unit of $\mathrm{T}_{5}{ }^{3-}$ unstable to electron loss and able to promptly tunnel through the repulsive Coulomb barrier are in the $2-50 \mathrm{~s}$ range, which is reasonably similar to what the Parks experiments see for -3 to -2 ion charge loss. For these reasons, we believe that the dynamical simulations and extrapolation techniques used in this work offer a good approach to better understanding the longtime dynamics of charge loss.

It might be surprising that the $\ln$ (rate) data seem to vary as $V$ and $V^{2}$ rather than linearly with $V$. We had expected a more linear variation because the activation energy $E$ is directly related to $V$. Nevertheless, all of our plots (i.e., for $q=0.3$ through $q$ $=1.0)$ of $\ln$ (rate) vs $V$ display strong quadratic character, so we believe this is no artifact. To understand the origin of the linear-plus-quadratic $V$ dependence, we consider the three negative phosphate units $(1,3$, and 5$)$ to be linked by harmonic "springs" as depicted in Figure 12. The springs represent the potential energy of the oligonucleotide's bond framework that constrains the three negative sites to undergo motions about their minimum-energy positions.

At the equilibrium geometry of the $\mathrm{T}_{5}{ }^{3-}$ ion, $r_{1,3}$ and $r_{3,5}$ have the values $r^{\mathrm{e}_{1,3}}$ and $r_{3,5}^{\mathrm{e}_{3}}$ and the total potential energy of the ion (i.e., the sum of its bond-stretch, torsional, van der Waals, Coulomb repulsion, and all other energies) is denoted $E^{\circ}$. As the distances $r_{1,3}$ and $r_{3,5}$ deviate from their equilibrium values, the total potential energy $E$ and the Coulomb potential $V(3)$ at the central phosphate site will vary in two qualitatively different ways. The Coulomb repulsions among the three negative sites will vary as $e^{2} /\left|r-r^{\mathrm{e}}\right| \approx e^{2} / r^{\mathrm{e}}\left[1-\left|r-r^{\mathrm{e}}\right| / r^{\mathrm{e}}+\ldots\right.$. Thus, the Coulomb potential at the central site $V$ will contain leading terms that vary linearly with the displacements $r-r^{\mathrm{e}}$. On the other hand, the total potential energy $E$ is expected, at least for small displacements around the equilibrium geometry, to vary as $E_{\text {bond }^{-}}$ $\left(r^{\mathrm{e}}\right)+a\left(r-r^{\mathrm{e}}\right)^{2}+\ldots$, that is, to have a lead term that is quadratic in $r-r^{\mathrm{e}}$. A movement of the three negative sites that causes $V$ to increase by a specified amount, $\delta V$, will correspond to displacements $\delta r$ that scale linearly with $\delta V$. However, these same displacements $\delta r$ will generate changes $\delta E$ in the total potential energy that depend quadratically on $\delta r$. It is likely for this reason that $\delta E$ seems to vary quadratically with $\delta V$, and as a result $\ln$ (rate) varies quadratically with $\delta V$.

\section{Summary}

On the basis of our molecular dynamics study of gas-phase oligonucleotides $\left(\mathrm{T}_{5}{ }^{3-}\right)$ containing five thymines, five deoxyri- boses, and five phosphates (three of which are negatively charged) at $T=170{ }^{\circ} \mathrm{C}$, we are able to make the following conclusions.

(1) $\mathrm{T}_{5}{ }^{3-}$ undergoes charge loss by electron ejection from its central phosphate site at a rate of $0.02-0.5 \mathrm{~s}^{-1}$ at $T=170{ }^{\circ} \mathrm{C}$.

(2) This electron ejection occurs when the repulsive Coulomb potential at the central phosphate fluctuates from its most probable value of ca. $4.2 \mathrm{eV}$ to ca. $5.1 \mathrm{eV}$, at which time the electron has enough energy to promptly tunnel through the Coulomb barrier.

(3) Our computed rates $\left(0.02-0.5 \mathrm{~s}^{-1}\right)$ and an activation energy of $0.9 \mathrm{eV}$ are consistent with a preexponential $A$ factor of $4 \times 10^{8}$ to $9 \times 10^{9} \mathrm{~s}^{-1}$.

(4) Electron ejection from the terminal phosphate sites is likely to be considerably slower than from the central phosphate because the Coulomb potentials at the terminal sites attain the $5.1 \mathrm{eV}$ critical value much less frequently. The primary reason underlying the reduced activity of the terminal sites lies in the simple geometrical fact that these sites are, on average, further from the other negative sites than is the central phosphate.

Although the $\mathrm{T}_{5}{ }^{3-}$ system studied here is not identical to one of the species examined in the Parks experiments, we believe it contains the most important features of all Parks species as far as the charge-loss process is concerned. Therefore, we feel confident in putting forth the following propositions concerning the charge-loss processes that have been experimentally observed.

(1) When multiply negatively charged oligonucleotides are sprayed from solution into the gas phase, only those ions containing no nearest-neighbor negative phosphate sites persist long enough to be observed on the time scales of the published Parks experiments. For example, species such as $\mathrm{T}_{5}{ }^{4-}$, ${\text { (ATATATA })^{5-} \text {, or (ATATATA) }}^{6-}$ do not persist long and thus are not observed, although experiments carried out on shorter time scales should be able to observe their decay dynamics. The reason they do not survive long in the gas phase is that the potential at the one phosphate site experiencing the highest Coulomb repulsion will exceed $5.1 \mathrm{eV}^{8}$ very soon after the gas phase is reached. It is on the basis of such considerations that we believe the only negative phosphate sites occurring in the samples probed in the Parks experiments are not nearest neighbors to any other such site.

(2) The structures realized in the gas phase for the highest charged species (e.g., $\mathrm{T}_{5}{ }^{3-}$ or (ATATATA) ${ }^{4-}$ ) are, on average, rather extended because of the Coulomb repulsions among their anion centers. This causes the phosphate sites nearer the termini to experience lower Coulomb repulsions than those more toward the interior. In fact, it is the most "central" phosphate site that will, on average, have the highest Coulomb potential and thus be most susceptible to charge loss.

(3) The charge-loss process occurs when structural dynamical fluctuations cause fluctuations in the Coulomb potential at a phosphate site of sufficient magnitude to render that site capable of prompt electron detachment (i.e., when the potential exceeds $5.1 \mathrm{eV}$ ). The detachment will be "prompt" when the tunneling rate exceeds the rate at which fluctuations bring the Coulomb potential from its most probable value (which we found to be ca. $4.2 \mathrm{eV}$ for $\mathrm{T}_{5}{ }^{3-}$ ) to $5.1 \mathrm{eV}$, at which time the electron can tunnel quickly outward.

(4) Once a $-n$ charged anion has undergone charge loss to form a $-(n-1)$ charged species, subsequent charge loss to form a $-(n-2)$ charged species will be slower because the most probable Coulomb potentials at each remaining anion site will be lower than in the $-n$ species. For example, the rate of 
going from $\mathrm{T}_{5}{ }^{4-}$ to $\mathrm{T}_{5}{ }^{3-}$ will be faster than for going from $\mathrm{T}_{5}{ }^{3-}$ to $\mathrm{T}_{5}{ }^{2-}$, which, in turn, will be faster than that for $\mathrm{T}_{5}{ }^{2-}$ going to $\mathrm{T}_{5}{ }^{-1}$. Our simulations have not yet verified this particular proposition, but we are presently carrying out simulations on such a system. We are also in the process of carrying out simulations at different temperatures to further test whether the $22-23 \mathrm{kcal} \mathrm{mol}^{-1}$ activation energy extracted from the experimental data can be reproduced by simulations using our model of the detachment process.

Acknowledgment. This work was supported by NSF Grant Nos. 9982420 and 0240387 to J.S. and by the Polish State Committee for Scientific Research (KBN), Grant No. DS/83714-0137-4 to P.S. Significant computer time provided by the Center for High Performance Computing at the University of Utah and by the Academic Computer Center in Gdansk (TASK) is also gratefully acknowledged. Finally, we thank Dr. Jacek Jakowski for assisting us in the early stages of our considerations of these problems and Dr. Joel Parks for much helpful input and for nurturing our interest in the processes treated here.

\section{References and Notes}

(1) Danell, A. S.; Parks, J. H. J. Am. Soc. Mass Spectrom. 2003, 14, $1330-1339$.
(2) These species consist of DNA bases connected to deoxyribose groups which, in turn, are bonded to phosphate groups. The ions are introduced into the gas phase by spraying samples that, in their liquid phase, likely have all of the phosphate groups negatively charged. However, once in the gas phase, the sample loses electrons (probably from one or more of the phosphate groups) to produce an anion of lower overall charge than the number of phosphate units it contains.

(3) The charge loss has been determined to arise from electron ejection rather than molecular fragmentation because the charge-to-mass ratio of the ions produced when the charge is lowered is consistent with no mass loss.

(4) Fluorescent labels are used to introduce excess energy into the gasphase ions in some of the Parks experiments. However, the charge-loss phenomenon that we deal with in this paper occurs with or without the label.

(5) Simons, J.; Skurski, P.; Barrios, R. J. Am. Chem. Soc. 2000, 122, 11893-11899.

(6) Case, D. A.; Pearlman, D. A.; Caldwell, J. W.; Cheatham, T. E., III; Wang, J.; Ross, W. S.; Simmerling, C. L.; Darden, T. A.; Merz, K. M.; Stanton, R. V.; Cheng, A. L.; Vincent, J. J.; Crowley, M.; Tsui, V.; Gohlke, H.; Radmer, R. J.; Duan, Y.; Pitera, J.; Massova, I.; Seibel, G. L.; Singh, U. C.; Weiner, P. K.; Kollman, P. A. AMBER 7, University of California, San Francisco, 2002.

(7) Boyly, C. I.; Cieplak, P.; Cornell, W. D.; Kollman, P. A. J. Phys. Chem. 1993, 97, 10269-10280.

(8) Two phosphate units that are nearest neighbors have most-probable distances of approximately $4 \AA$ (and can fluctuate to even closer distances) and thus produce Coulomb repulsions of $14.4 / 4=3.6 \mathrm{eV}$. When the Coulomb interactions of any other negative sites are added to this, the total potential easily exceeds $5.1 \mathrm{eV}$. 